\title{
Asymptotic entanglement in open quantum systems
}

\author{
Aurelian Isar \\ Department of Theoretical Physics, National Institute of Physics and Nuclear Engineering \\ Bucharest-Magurele, Romania \\ isar@theory.nipne.ro
}

\begin{abstract}
In the framework of the theory of open systems based on completely positive quantum dynamical semigroups, we solve in the asymptotic long-time regime the master equation for two independent harmonic oscillators interacting with an environment. We give a description of the continuous-variable asymptotic entanglement in terms of the covariance matrix of the considered subsystem for an arbitrary Gaussian input state. Using Peres-Simon necessary and sufficient condition for separability of twomode Gaussian states, we show that for certain classes of environments the initial state evolves asymptotically to an entangled equilibrium bipartite state, while for other values of the coefficients describing the environment, the asymptotic state is separable. We calculate also the logarithmic negativity characterizing the degree of entanglement of the asymptotic state.
\end{abstract}

\section{Introduction}

When two systems are immersed in an environment, then, besides and at the same time with the quantum decoherence phenomenon, the environment can also generate a quantum entanglement of the two systems [1,2]. In certain circumstances, the environment enhances the entanglement and in others it suppresses the entanglement and the state describing the two systems becomes separable. The structure of the environment may be such that not only the two systems become entangled, but also such that the entanglement is maintained for a definite time or a certain amount of entanglement survives in the asymptotic long-time regime.

In the present paper we investigate, in the framework of the theory of open systems based on completely positive quantum dynamical semigroups, the existence of the continuous variable asymptotic entanglement for a subsystem composed of two identical harmonic oscillators interacting with an environment. We are interested in discussing the correlation effect of the environment, therefore we assume that the two systems are independent, i.e. they do not interact directly. The initial state of the subsystem is taken of Gaussian form and the evolution under the quantum dynamical semigroup assures the preservation in time of the Gaussian form of the state. We only investigate here the asymptotic behaviour of the subsystem states. The time evolution of the entanglement, in particular the possibility of the so-called "entanglement sudden death", that is suppression of the entanglement at a certain finite moment of time, will be discussed in a future work.

The organizing of the paper is as follows. In Sec. 2 we write the equations of motion in the Heisenberg picture for two independent harmonic oscillators interacting with a general 
environment. With these equations we derive in Sec. 3 the asymptotic values of variances and covariances of coordinates and momenta which enter the asymptotic covariance matrix. Then, by using the Peres-Simon necessary and sufficient condition for separability of twomode Gaussian states [3, 4], we investigate the behaviour of the environment induced entanglement in the limit of long times. We show that for certain classes of environments the initial state evolves asymptotically to an equilibrium state which is entangled, while for other values of the parameters describing the environment, the entanglement is suppressed and the asymptotic state is separable. The existence of the quantum correlations between the two systems in the asymptotic long-time regime is the result of the competition between entanglement and quantum decoherence. A summary is given in Sec. 4.

\section{Equations of motion for two independent harmonic oscillators}

We study the dynamics of the subsystem composed of two identical non-interacting oscillators in weak interaction with an environment. In the axiomatic formalism based on completely positive quantum dynamical semigroups, the irreversible time evolution of an open system is described by the following general quantum Markovian master equation for an operator $A$ (Heisenberg representation) [5, 6]:

$$
\frac{d A(t)}{d t}=\frac{i}{\hbar}[H, A(t)]+\frac{1}{2 \hbar} \sum_{j}\left(V_{j}^{\dagger}\left[A(t), V_{j}\right]+\left[V_{j}^{\dagger}, A(t)\right] V_{j}\right) .
$$

Here, $H$ denotes the Hamiltonian of the open system and $V_{j}, V_{j}^{\dagger}$ which are operators defined on the Hilbert space of $H$, represent the interaction of the open system with the environment. Being interested in the set of Gaussian states, we introduce those quantum dynamical semigroups that preserve that set. Therefore $H$ is taken to be a polynomial of second degree in the coordinates $x, y$ and momenta $p_{x}, p_{y}$ of the two quantum oscillators and $V_{j}, V_{j}^{\dagger}$ are taken polynomials of only first degree in these canonical observables. Then in the linear space spanned by the coordinates and momenta there exist only four linearly independent operators $V_{j=1,2,3,4}$ [7] :

$$
V_{j}=a_{x j} p_{x}+a_{y j} p_{y}+b_{x j} x+b_{y j} y,
$$

where $a_{x j}, a_{y j}, b_{x j}, b_{y j} \in \mathbf{C}$. The Hamiltonian $H$ of the two uncoupled identical harmonic oscillators of mass $m$ and frequency $\omega$ is given by

$$
H=\frac{1}{2 m}\left(p_{x}^{2}+p_{y}^{2}\right)+\frac{m \omega^{2}}{2}\left(x^{2}+y^{2}\right) .
$$

The fact that the evolution is given by a dynamical semigroup implies the positivity of the following matrix formed by the scalar products of the four vectors $\mathbf{a}_{x}, \mathbf{b}_{x}, \mathbf{a}_{y}, \mathbf{b}_{y}$ whose 
entries are the components $a_{x j}, b_{x j}, a_{y j}, b_{y j}$, respectively:

$$
\frac{1}{2} \hbar\left(\begin{array}{cccc}
\left.\mathbf{a}_{x} \mathbf{a}_{x}\right) & \left(\mathbf{a}_{x} \mathbf{b}_{x}\right) & \left(\mathbf{a}_{x} \mathbf{a}_{y}\right) & \left(\mathbf{a}_{x} \mathbf{b}_{y}\right) \\
\left(\mathbf{b}_{x} \mathbf{a}_{x}\right) & \left(\mathbf{b}_{x} \mathbf{b}_{x}\right) & \left(\mathbf{b}_{x} \mathbf{a}_{y}\right) & \left(\mathbf{b}_{x} \mathbf{b}_{y}\right) \\
\left(\mathbf{a}_{y} \mathbf{a}_{x}\right) & \left(\mathbf{a}_{y} \mathbf{b}_{x}\right) & \left(\mathbf{a}_{y} \mathbf{a}_{y}\right) & \left(\mathbf{a}_{y} \mathbf{b}_{y}\right) \\
\left(\mathbf{b}_{y} \mathbf{a}_{x}\right) & \left(\mathbf{b}_{y} \mathbf{b}_{x}\right) & \left(\mathbf{b}_{y} \mathbf{a}_{y}\right) & \left(\mathbf{b}_{y} \mathbf{b}_{y}\right.
\end{array}\right)
$$

For simplicity we take this matrix of the following form, where all coefficients $D_{x x}, D_{x p_{x}}, \ldots$ and $\lambda$ are real quantities:

$$
\left(\begin{array}{cccc}
D_{x x} & -D_{x p_{x}}-i \hbar \lambda / 2 & D_{x y} & -D_{x p_{y}} \\
-D_{x p_{x}}+i \hbar \lambda / 2 & D_{p_{x} p_{x}} & -D_{y p_{x}} & D_{p_{x} p_{y}} \\
D_{x y} & -D_{y p_{x}} & D_{y y} & -D_{y p_{y}}-i \hbar \lambda / 2 \\
-D_{x p_{y}} & D_{p_{x} p_{y}} & -D_{y p_{y}}+i \hbar \lambda / 2 & D_{p_{y} p_{y}}
\end{array}\right) .
$$

It follows that the principal minors of this matrix are positive or zero. From the CauchySchwarz inequality the following relations for the coefficients defined in Eq. (5) hold (from now on we put, for simplicity, $\hbar=1$ ):

$$
\begin{gathered}
D_{x x} D_{y y}-D_{x y}^{2} \geq 0, D_{x x} D_{p_{x} p_{x}}-D_{x p_{x}}^{2} \geq \frac{\lambda^{2}}{4}, D_{x x} D_{p_{y} p_{y}}-D_{x p_{y}}^{2} \geq 0, \\
D_{y y} D_{p_{x} p_{x}}-D_{y p_{x}}^{2} \geq 0, D_{y y} D_{p_{y} p_{y}}-D_{y p_{y}}^{2} \geq \frac{\lambda^{2}}{4}, D_{p_{x} p_{x}} D_{p_{y} p_{y}}-D_{p_{x} p_{y}}^{2} \geq 0 .
\end{gathered}
$$

The covariance of operators $A_{1}$ and $A_{2}$ can be written with the density operator $\rho$, describing the initial state of the quantum system, as follows:

$$
\sigma_{A_{1} A_{2}}(t)=\frac{1}{2} \operatorname{Tr}\left(\rho\left(A_{1} A_{2}+A_{2} A_{1}\right)(t)\right) .
$$

We introduce the following $4 \times 4$ bimodal covariance matrix:

$$
\sigma(t)=\left(\begin{array}{cccc}
\sigma_{x x} & \sigma_{x p_{x}} & \sigma_{x y} & \sigma_{x p_{y}} \\
\sigma_{x p_{x}} & \sigma_{p_{x} p_{x}} & \sigma_{y p_{x}} & \sigma_{p_{x} p_{y}} \\
\sigma_{x y} & \sigma_{y p_{x}} & \sigma_{y y} & \sigma_{y p_{y}} \\
\sigma_{x p_{y}} & \sigma_{p_{x} p_{y}} & \sigma_{y p_{y}} & \sigma_{p_{y} p_{y}}
\end{array}\right)
$$

By direct calculation we obtain [7] ( $\mathrm{T}$ denotes the transposed matrix):

$$
\frac{d \sigma}{d t}=Y \sigma+\sigma Y^{\mathrm{T}}+2 D
$$

where

$$
Y=\left(\begin{array}{cccc}
-\lambda & 1 / m & 0 & 0 \\
-m \omega^{2} & -\lambda & 0 & 0 \\
0 & 0 & -\lambda & 1 / m \\
0 & 0 & -m \omega^{2} & -\lambda
\end{array}\right), \quad D=\left(\begin{array}{cccc}
D_{x x} & D_{x p_{x}} & D_{x y} & D_{x p_{y}} \\
D_{x p_{x}} & D_{p_{x} p_{x}} & D_{y p_{x}} & D_{p_{x} p_{y}} \\
D_{x y} & D_{y p_{x}} & D_{y y} & D_{y p_{y}} \\
D_{x p_{y}} & D_{p_{x} p_{y}} & D_{y p_{y}} & D_{p_{y} p_{y}}
\end{array}\right) .
$$


The time-dependent solution of Eq. (9) is given by [7]

$$
\sigma(t)=M(t)(\sigma(0)-\sigma(\infty)) M^{\mathrm{T}}(t)+\sigma(\infty),
$$

where $M(t)=\exp (Y t)$. The matrix $M(t)$ has to fulfil the condition $\lim _{t \rightarrow \infty} M(t)=0$. In order that this limit exists, $Y$ must only have eigenvalues with negative real parts. The values at infinity are obtained from the equation [7]

$$
Y \sigma(\infty)+\sigma(\infty) Y^{\mathrm{T}}=-2 D .
$$

\section{Environment induced entanglement}

The two-mode Gaussian state is entirely specified by its covariance matrix $\sigma$ (8) , which is a real, symmetric and positive matrix with the following block structure:

$$
\sigma=\left(\begin{array}{cc}
A & C \\
C^{\mathrm{T}} & B
\end{array}\right)
$$

where $A, B$ and $C$ are $2 \times 2$ matrices. Their entries are correlations of the canonical operators $x, y, p_{x}, p_{y}, A$ and $B$ denote the symmetric covariance matrices for the individual reduced one-mode states, while the matrix $C$ contains the cross-correlations between modes. In the following we shall consider environments for which $D_{x x}=D_{y y}, D_{x p_{x}}=$ $D_{y p_{y}}, D_{p_{x} p_{x}}=D_{p_{y} p_{y}}, D_{x p_{y}}=D_{y p_{x}}$. Then both unimodal covariance matrices are equal, $A=B$ and the entanglement matrix $C$ is symmetric. With the chosen coefficients, we obtain from Eq. (12) the following elements of the asymptotic entanglement matrix $C$ :

$$
\begin{gathered}
\sigma_{x y}(\infty)=\frac{m^{2}\left(2 \lambda^{2}+\omega^{2}\right) D_{x y}+2 m \lambda D_{x p_{y}}+D_{p_{x} p_{y}}}{2 m^{2} \lambda\left(\lambda^{2}+\omega^{2}\right)}, \\
\sigma_{x p_{y}}(\infty)=\sigma_{y p_{x}}(\infty)=\frac{-m^{2} \omega^{2} D_{x y}+2 m \lambda D_{x p_{y}}+D_{p_{x} p_{y}}}{2 m\left(\lambda^{2}+\omega^{2}\right)}, \\
\sigma_{p_{x} p_{y}}(\infty)=\frac{m^{2} \omega^{4} D_{x y}-2 m \omega^{2} \lambda D_{x p_{y}}+\left(2 \lambda^{2}+\omega^{2}\right) D_{p_{x} p_{y}}}{2 \lambda\left(\lambda^{2}+\omega^{2}\right)} .
\end{gathered}
$$

The elements of matrices $A$ and $B$ are obtained by taking $x=y$ in the previous expressions. We calculate the determinant of the entanglement matrix and obtain:

$$
\operatorname{det} C=\frac{1}{4 \lambda^{2}\left(\lambda^{2}+\omega^{2}\right)}\left[\left(m \omega^{2} D_{x y}+\frac{1}{m} D_{p_{x} p_{y}}\right)^{2}+4 \lambda^{2}\left(D_{x y} D_{p_{x} p_{y}}-D_{x p_{y}}^{2}\right)\right] .
$$

Gaussian states with $\operatorname{det} C \geq 0$ are separable states, but for $\operatorname{det} C<0$ it may be possible that the asymptotic equilibrium states are entangled. In order to investigate whether the 
environment can actually entangle the two independent systems, we can use the partial transposition criterion [3, 4]: a state is entangled if and only if the operation of partial transposition does not preserve its positivity. Simon [4] obtained the following necessary and sufficient criterion for separability: $S \geq 0$, where

$$
S \equiv \operatorname{det} A \operatorname{det} B+\left(\frac{1}{4}-|\operatorname{det} C|\right)^{2}-\operatorname{Tr}\left[A J C J B J C^{\mathrm{T}} J\right]-\frac{1}{4}(\operatorname{det} A+\operatorname{det} B)
$$

and $J$ is the $2 \times 2$ symplectic matrix.

In order to analyze the possible persistence of the environment induced entanglement in the asymptotic long-time regime, we consider the environment characterized by the following values of its parameters: $m^{2} \omega^{2} D_{x x}=D_{p_{x} p_{x}}, \quad D_{x p_{x}}=0, m^{2} \omega^{2} D_{x y}=D_{p_{x} p_{y}}$. In this case the Simon expression (18) takes the form:

$$
S=\left(\frac{m^{2} \omega^{2}\left(D_{x x}^{2}-D_{x y}^{2}\right)}{\lambda^{2}}+\frac{D_{x p_{y}}^{2}}{\lambda^{2}+\omega^{2}}-\frac{1}{4}\right)^{2}-4 \frac{m^{2} \omega^{2} D_{x x}^{2} D_{x p_{y}}^{2}}{\lambda^{2}\left(\lambda^{2}+\omega^{2}\right)} .
$$

For environments characterized by such coefficients that the expression (19) is strictly negative, the asymptotic final state is entangled. In particular, if $D_{x y}=0$, we obtain that $S<0$, i.e. the asymptotic final state is entangled, for the following range of values of the coefficient $D_{x p_{y}}$ characterizing the environment [8]:

$$
\frac{m \omega D_{x x}}{\lambda}-\frac{1}{2}<\frac{D_{x p_{y}}}{\sqrt{\lambda^{2}+\omega^{2}}}<\frac{m \omega D_{x x}}{\lambda}+\frac{1}{2}
$$

where the coefficient $D_{x x}$ satisfies the condition $m \omega D_{x x} / \lambda \geq 1 / 2$, equivalent with the unimodal uncertainty relation. If the coefficients do not fulfil the inequalities (20), then $S \geq 0$ and the asymptotic state of the considered system is separable.

We apply the measure of entanglement based on negative eigenvalues of the partial transpose of the subsystem density matrix. For a Gaussian density operator, the negativity is completely defined by the symplectic spectrum of the partial transpose of the covariance matrix. The logarithmic negativity $E=-\frac{1}{2} \log _{2}[4 f(\sigma)]$ determines the strength of entanglement for $E>0$. If $E \leq 0$, then the state is separable. Here

$$
f(\sigma)=\frac{1}{2}(\operatorname{det} A+\operatorname{det} B)-\operatorname{det} C-\sqrt{\left[\frac{1}{2}(\operatorname{det} A+\operatorname{det} B)-\operatorname{det} C\right]^{2}-\operatorname{det} \sigma .}
$$

In our case the logarithmic negativity has the form

$$
E=-\log _{2}\left[2\left|\frac{m \omega D_{x x}}{\lambda}-\frac{D_{x p_{y}}}{\sqrt{\lambda^{2}+\omega^{2}}}\right|\right] .
$$

It depends only on the diffusion and dissipation coefficients characterizing the environment and does not depend on the initial Gaussian state. In Fig. 1 logarithmic negativity $E$ is represented versus scaled diffusion coefficients $D_{x x}$ and $D_{x p_{y}}$. 


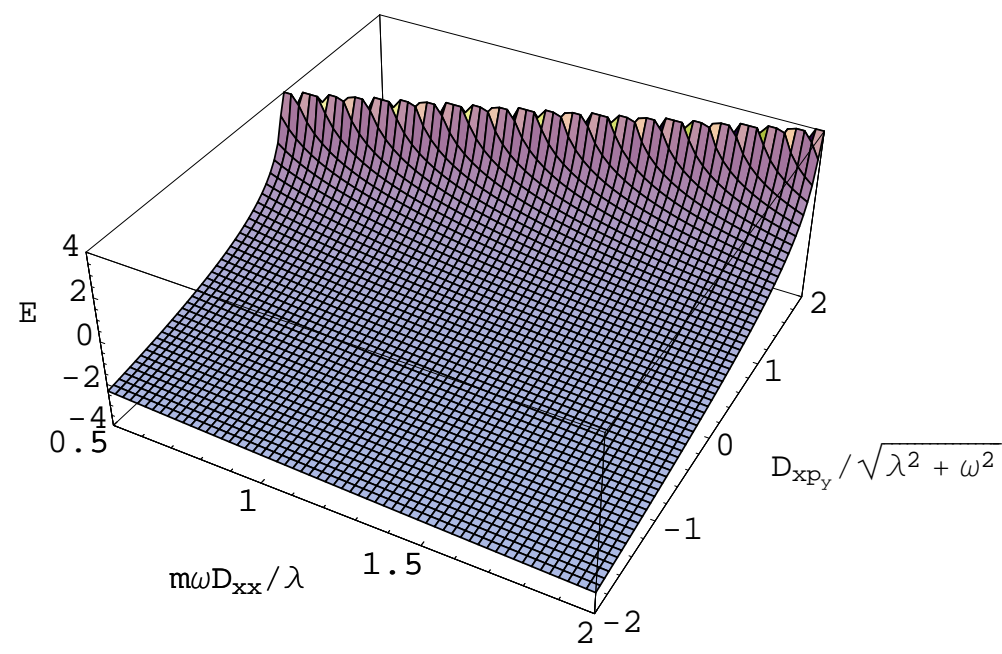

Figure 1: Logarithmic negativity versus scaled diffusion coefficients.

\section{Summary}

In the framework of the theory of open quantum systems based on completely positive quantum dynamical semigroups, we investigated the existence of the asymptotic quantum entanglement for a subsystem composed of two uncoupled identical harmonic oscillators interacting with an environment. By using the Peres-Simon necessary and sufficient condition for separability of two-mode Gaussian states, we have shown that for certain classes of environments the initial state evolves asymptotically to an equilibrium state which is entangled, i.e. there exist non-local quantum correlations for the bipartite states of the two harmonic oscillator subsystem, while for other values of the coefficients describing the environment, the asymptotic state is separable. We determined also the logarithmic negativity characterizing the degree of entanglement of the asymptotic state. Due to the increased interest manifested towards the continuous variables approach to quantum information theory, these results, in particular the possibility of maintaining a bipartite entanglement in a diffusive-dissipative environment for asymptotic long times, might be useful for applications in the field of quantum information processing and communication.

\section{Acknowledgments}

The author acknowledges the financial support received within the Project CEEX 68/2005. 


\section{References}

[1] F. Benatti, R. Floreanini, Int. J. Mod. Phys. B 19 (2005) 3063-3139.

[2] F. Benatti, R. Floreanini, J. Phys. A: Math. Gen. 39 (2006) 2689-2699.

[3] A. Peres, Phys. Rev. Lett. 77 (1996) 1413-1415.

[4] R. Simon, Phys. Rev. Lett. 84 (2000) 2726-2729.

[5] G. Lindblad, Commun. Math. Phys. 48 (1976) 119-130.

[6] A. Isar, A. Sandulescu, H. Scutaru, E. Stefanescu, W. Scheid, Int. J. Mod. Phys. E 3 (1994) 635-714.

[7] A. Sandulescu, H. Scutaru, W. Scheid, J. Phys. A: Math. Gen. 20 (1987) 2121-2131.

[8] A. Isar, J. Russ. Laser Res. 28 (2007) 439-452. 